\title{
LA METROVÍA COMO SISTEMA BRT - EL CASO DE LA CIUDAD DE GUAYAQUIL
}

\section{THE METROVIA AS A BRT SYSTEM - THE CASE OF THE CITY OF GUAYAQUIL}

\author{
NARANJO RAMOS, Yelitza \\ Universidad Católica de Santiago de Guayaquil (UCSG) \\ Facultad Arquitectura y Diseño, Instituto de Planificación Urbana y Regional (IPUR) \\ Docente Titular, Coordinador Académico 1 \\ Urdenor 1 mz 138, v 13, Guayaquil, Guayas, Ecuador \\ Correo electrónico: yelitza 24@hotmail.com \\ Teléfono: +593 (04) 2222025 / +593 (04) 2222024
}

\author{
ARELLANO, Blanca \\ Universidad Politécnica de Cataluña (UPC) \\ Departamento de Tecnología de la Arquitectura (TA), Centro de Política de Suelo y Valoraciones (CPSV) \\ Profesora asociada y Personal de Investigación (PSR) \\ Av. Diagonal 649, 08028 Barcelona, España \\ Correo electrónico: blanca.arellano@upc.edu \\ Teléfono: +34 934054383
}

Palabras Clave: Metrovía; Guayaquil; transporte público masivo

Key words: Metrovia; Guayaquil; mass public transport

\section{Resumen}

La Metrovía de Guayaquil es parte del nuevo sistema de transporte público masivo BRT, el mismo que se presentó como un transporte público masivo que combina estaciones, vehículos, planificación y elementos inteligentes en un sistema integral. Este sistema además involucra corredores de vías de autobuses en carriles segregados, ya sea a nivel o desnivel, así como tecnología de autobús modernizada, sin embargo, dentro del circuito en el que se desarrolla el sistema se evidencia la inexistencia de espacios flexibles y eficaces que impiden el desplazamiento en distancias cortas, es por esto que el uso del vehículo es excesivo dentro de la ciudad tanto en las calles y avenidas como en áreas libres para estacionamientos. Si bien las ciudades no mueren, si no que crecen y se transforman, como consecuencia de la economía local, las necesidades de sus habitantes y de la capacidad de respuesta de las entidades que tienen competencias sobre el mejoramiento de su calidad de vida. Producto de ese crecimiento, la ciudad ha buscado implantar un mecanismo que represente mayores beneficios en términos de tiempo, dinero y calidad del servicio, pero tal vez dejando de lado el estudio de esas particularidades que denotan un respeto por el entorno construido.

En Guayaquil uno de los puntos principales es que el centro de la ciudad posee diversos anchos en sus ejes viales que están en algunos casos entre 11 y 18 metros, estas calles, no han sido diseñadas para este sistema de transporte donde además de ocupar los carriles mixtos (vehículos privados, taxis, motos y transporte público) se coloca un elemento central que en este caso son las estaciones de buses. Por ello el objetivo central es identificar como 
este sistema ha sido parte de la estructura urbana de la ciudad, adicionalmente se busca identificar si es un proyecto de movilidad sostenible y de diversidad en el suelo de la ciudad.

La metodología que se plantea es a través de encuestas realizada por el autor como finalidad conocer las diferentes actividades y percepción de los usuarios de la Metrovía, de tal manera que se identifique el uso del sistema, las paradas, troncales y ejes donde existe una movilidad mayor y la calidad de los espacios tanto a nivel de paradas, como de terminales y buses, otro punto es la observación participante u observación participativa que ha sido por muchos años parte del estudio de las ciudades, estudios cualitativos que se incluyen como una forma de recoger información, tales como entrevistas, observación y análisis de documentos.

\section{Abstract}

The Guayaquil Metro is part of the new BRT transport system, which was presented as a mass public transport that combines stations, vehicles, planning and intelligent elements in an integral system. This system also involves bus corridors in segregated lanes, either level or vertical, as well as modernized bus technology. However, within the circuit in which the system is developed, the inexistence of flexible and efficient spaces that prevent displacement over short distances is evident, which is why the use of the vehicle is excessive within the city, both on streets and avenues, in free areas for parking. Although cities do not die, they grow and transform, as a consequence of the local economy, the needs of their inhabitants and the capacity of response of the entities that have competences on the improvement of their quality of life. Product of that growth, the city has sought to implement a mechanism that represents greater benefits in terms of time, money and quality of service, but perhaps leaving aside the study of those particularities that denote a respect for the built environment.

In Guayaquil one of the main points is that the center of the city has different widths in its roads that are in some cases between 11 and 18 meters, these streets, have not been designed for this transport system where in addition to occupy the lanes mixed (private vehicles, taxis, motorcycles and public transport) is placed a central element that in this case are the bus stations. Therefore, the central objective in this research is to analyze the impact that has occurred in the urban structure of Guayaquil from the mass public transport system and how it has contributed to the improvement of the quality of life of the inhabitants of the city. Additionally, we seek to identify how mass public transport is a project of sustainable mobility and diversity in the city's soil.

The methodology proposed is through surveys conducted by the author as a purpose to know the different activities and perceptions of the users of the Metrovia, in such a way that the use of the system, stops, trunks and axes where there is mobility is identified greater and the quality of the spaces at the level of stops, terminals and buses, another point is participant observation or participatory observation that has been part of the study of cities for many years, qualitative studies that are included as a form of collect information, such as interviews, observation and analysis of documents.

\section{Introducción}

El transporte es uno de los temas más complicados de gestionar en una ciudad, un indicador de esta complejidad es el hecho de que dicho transporte, a diferencia de todos los demás 
componentes, no se mejora con el desempeño económico, sino que se agrava. En América Latina han tenido una tasa de urbanización creciente en las últimas décadas, y una consiguiente mayor demanda de transporte por parte de los ciudadanos. Las ciudades se han vuelto un eje fundamental del desarrollo económico de un país, y sus sistemas de transporte son uno de los componentes más importantes de este desarrollo (Rogat, 2009).

Las grandes ciudades del mundo que han resuelto su problema de transporte público lo han logrado mediante una expansión de la red de Metro, trenes urbanos y una regulación en el uso del automóvil. La evidencia a nivel mundial ha permitido constatar que la expansión de redes de Metro genera una reducción más efectiva en el uso del transporte privado que otras medidas, como por ejemplo subsidiar la tarifa del transporte público. Adicionalmente, la existencia de una importante red de Metro o trenes urbanos permite aumentar notablemente la efectividad de políticas regulatorias al uso del automóvil, como tarificación vial y gravámenes específicos, entre otras, ya que los automovilistas habituales sí ven en el Metro una alternativa real de transporte, no así en los servicios de buses (De Grange C, 2010).

Guayaquil una ciudad ubicada a orillas del río Guayas, desarrollada en sentido norte - sur, aprovechaba la facilidad de comunicación que daba al mismo, es así que se ligaba directamente al sistema de transporte que en principio se daba mediante el sistema fluvial balsas, balandras y navíos para salir al mar. A finales del siglo pasado se organizó el transporte urbano mediante carros tirados por mulas que corrían sobre rieles por las pocas calles que se prestaban para ese tránsito.

Este transporte fue remplazado por tranvías eléctricos con recorrido principalmente de nortesur, que sirvieron hasta la década de los 40 cuando ya no pudieron competir con las líneas de autobuses. Este sistema de autobuses utilizaba vehículos de mediana capacidad, con motores a gasolina y con recorridos por las calles pavimentadas que existían.

El puerto marítimo al sur y el puente sobre el río guayas, al norte, ocasionaba que las mercaderías y el tránsito extra pesado recorra la zona céntrica de la ciudad, así como la ubicación norte del aeropuerto y Terminal Terrestre que obligaba a los usuarios del sur a cruzar toda la ciudad (Memorias, Guayaquil 2000, 1988).

Todos estos sistemas de transporte han generado cambios en la ciudad a lo largo del tiempo tanto a nivel físico como a nivel operacional, intentando solucionar problemas de congestión, accesibilidad a las diferentes zonas y confort. Es así como en los últimos años la sostenibilidad se ha implementado como una nueva variable a utilizarse dentro de los sistemas de transportes beneficiando no solo al medio ambiente sino también a la población.

\subsection{Transporte e Imagen Urbana a través de los años en la ciudad de Guayaquil}

El transporte urbano ha estado íntimamente ligado al crecimiento de la ciudad desde hace 115 años, a raíz del incremento de su población y de su economía a fines del siglo XIX, con el auge cacaotero. Esos primeros carros urbanos que tirados por mulas entraron a funcionar en $1880 \mathrm{y}$ en 1884 circulaban por el Malecón (Estrada Icaza, 1995).

A lo largo de los ejes viales las viviendas y edificios de la época eran parte de este atractivo recorrido que generaban los servicios de transporte, la relación directa que se da con las 
viviendas, la infraestructura, el entorno urbano y a su vez la presencia de vegetación en las aceras de las diferentes vías.

En 1885 se crea la empresa de Carros Urbano; en 1907, la ciudad contaba con 56 líneas de carros urbanos que recorrían 33.000 metros, el tren rotatorio de la empresa estaba constituido por 15 carros "imperiales" (diferenciados de los demás por poseer dos pisos), 6 "jardines", 10 mixtos, 12 cerrados, 6 "góndolas" (llamados así por no tener techo), 3 carrozas y 20 carros para carga, lo que sumaba 72 vehículos que conducían diariamente a un promedio de 20.000 pasajeros. Para ese entonces las principales líneas existentes en la ciudad eran las del Malecón, Astillero, Matadero, Victoria, Cementerio, Salado, Morro, Chanduy, Hipódromo y Luque, era en la ciudad el lugar perfecto para invertir en este negocio. Pero el tiempo pasó y la empresa comenzó a tener problemas, principalmente por no haber aceptado en su momento la implementación de los tranvías eléctricos (Hoyos G, 2008).

A principios del siglo XX aparece el tranvía eléctrico y ofrecía mayor comodidad. A pesar de los diferentes sistemas de transporte que hasta la época se venían incorporando de la misma manera cambiaban, sin embargo, la ciudad seguía manteniendo sus principales características y sus principales servicios administrativos, la accesibilidad, su relación directa al entorno natural, social y al río.

En 1940 creció el parque automotor de buses; en 1947 aparecieron los colectivos la primera versión fue los Station Wagon en que se iban seis personas sentadas y el pasaje costaba un sucre. Como la demanda aumentó se adaptaron carrocerías para convertirlos en vehículos tipo micro para 16 personas. (Vega \& Díaz, 2012).

En 1950 ya hubo 145 unidades, el crecimiento de los buses y colectivos no fue igual. En la década del cincuenta los colectivos aumentaron con relación a los buses y en los años 60 se seguía dando el mismo aumento de los colectivos y los buses a su vez disminuyeron (Vega \& Díaz, 2012).

En la década del 50 se empezaron a desarrollar nuevas urbanizaciones en la ciudad, debido al crecimiento poblacional que se generaba, todo esto ocasionó que aumentara el transporte para poder abastecer a la población sobre todo en los asentamientos populares que existían hasta esa época.

En los años 60 se trasladaron del Malecón y su centro urbano las principales actividades portuarias al nuevo puerto al sur de la ciudad, la ejecución de los puentes que unieron Guayaquil con el resto del país, fueron algunos hechos fundamentales que asociados a la falta de planificación urbanística dieron inicio al proceso de degeneración del centro urbano (Dreher A, 2009).

Es a partir de entonces que la ciudad comienza a darle la espalda a uno de sus recursos más importantes: el Río Guayas. Paralelamente se produjo el abandono del centro urbano como zona residencial, además de una intensa densificación comercial, limitando su uso al comercio y oficinas.

En 1975 ya existían 27 rutas de buses y diez años después era el parque automotor más alto de la ciudad (Vega \& Díaz, 2012). La ciudad de Guayaquil y su centro urbano estuvo sometida a un proceso de densificación comercial a partir de la década de los sesenta, previamente su relación con el río Guayas, razón fundamental de su creación y existencia, había dejado de ser 
vital por la disminución de las actividades económicas que tradicionalmente se desarrollaban en sus orillas.

Este hecho anterior, asociado a una creciente migración de los residentes hacia los nuevos barrios residenciales desarrollados en la periferia sentó las bases para un deterioro del centro de la ciudad, limitándose en gran parte a las actividades comerciales, de oficinas es decir actividades bancarias y públicas. Ante esta inevitable situación de deterioro, se planearon nuevos proyectos para la ciudad, uno de los principales fue el Proyecto Malecón 2000 que formaba parte de una regeneración urbana, generando nuevamente valor en el suelo aledaño al mismo y por consiguiente una considerable atracción de la inversión inmobiliaria que en ese momento estaba focalizada en otras áreas de la ciudad.

En 1999 se inauguró la primera etapa del Malecón; a lo largo del año 2000 - 2002 se inauguran las dos etapas restantes del malecón. En el 2006 el Municipio de Guayaquil, implementó el Sistema Integral de Transporte Masivo Urbano "Metrovía".

La ciudad empezaba una vez más a ser sometida a transformaciones físicas, en este caso con la implementación del nuevo sistema de transporte urbano masivo, que en principio corresponde a un sistema BRT (Bus Rapid Transit) presentado con beneficios sostenibles, es decir reducción de congestionamiento vehicular, movimiento de mayor cantidad de pasajeros y en menor tiempo que los buses urbanos anteriores, reducción de la contaminación ambiental, y confort en su desplazamiento.

\subsection{Estructura del sistema de transporte de personas en Guayaquil}

Guayaquil con una superficie de 33,310 Ha. (19,500 Ha. urbanizadas), posee una población de $2,644.145$ de habitantes con una tasa de crecimiento poblacional anual de $2.15 \%$, y una tasa de inflación: 3.36\% (Plan de Movilidad, 2013).

EI INEC en sus estadísticas menciona que en los 4 últimos años el medio más utilizado por los hogares es el transporte público (ver gráfico 1), sin embargo, se evidencia en las variaciones que ha tenido cada uno de los medios de transporte que se realizan a nivel nacional, una disminución desde el 2012 hasta el 2015 del transporte público en casi un 10\%, esto se debe a la demanda de vehículo privado que ha ido en aumento (INEC, Instituto Nacional de Estadística y Censos, 2014). Cabe mencionar que está gráfica es a nivel del Ecuador y toma datos de todas las ciudades.

\section{Gráfico 1. Medio para traslado a nivel nacional (\%)}

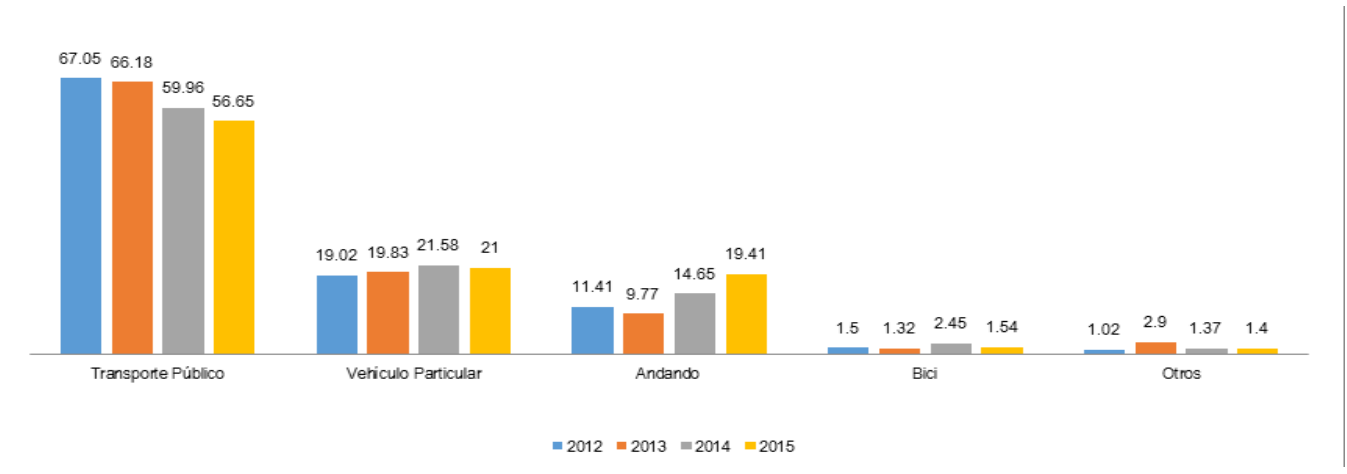

Fuente: (INEC, Instituto Nacional de Estadística y Censos, 2014)

Nota: Otros, se refiere a canoa, animales.

Citación: NARANJO RAMOS, Y. y ARELLANO, B. La Metrovía como sistema BRT - el caso de la ciudad de Guayaquil. En: Libro de proceedings, CTV 2018. XII Congreso Internacional Ciudad y Territorio Virtual. "Ciudades y Territorios Inteligentes". UNCuyo, Mendoza, 5-7 septiembre 2018. Barcelona: CPSV, 2018, p. 129-142. 


\subsubsection{Transporte público}

\section{- Masivo (Sistema Metrovía)}

Está conformado por las rutas, terminales, paradas, infraestructura y equipos incorporados al referido Sistema. Cuenta con 3 troncales, 4 terminales, 35 rutas alimentadoras que llegan a la troncal principal y 90 estaciones en varios sectores de la ciudad.

El costo del pasaje para el público en general es de $\$ 0.30$. Personas con capacidades especiales, estudiantes y personas de la tercera edad pagan $\$ 0.12$. También puede solicitar una tarjeta electrónica recargable para acceder a este servicio.

\section{- Urbano colectivo}

En cuanto al parque automotor de transporte público y vehículos livianos en Guayaquil, el Ing. Federico von Buchwald en su libro "Movilidad Urbana de Guayaquil", indica que entre los años 2003 y 2012 los vehículos de transporte público de capacidad menor tales como: furgonetas, microbuses y busetas, se redujeron convirtiéndose en buses de mayor capacidad, lo que redujo el número de vehículos de 9.216 a 4.455, optimizando el espacio por vehículo, al aumentar el número de buses pero en total reduciendo la capacidad de oferta.

Figura 1. Avenida Quito, año 2015

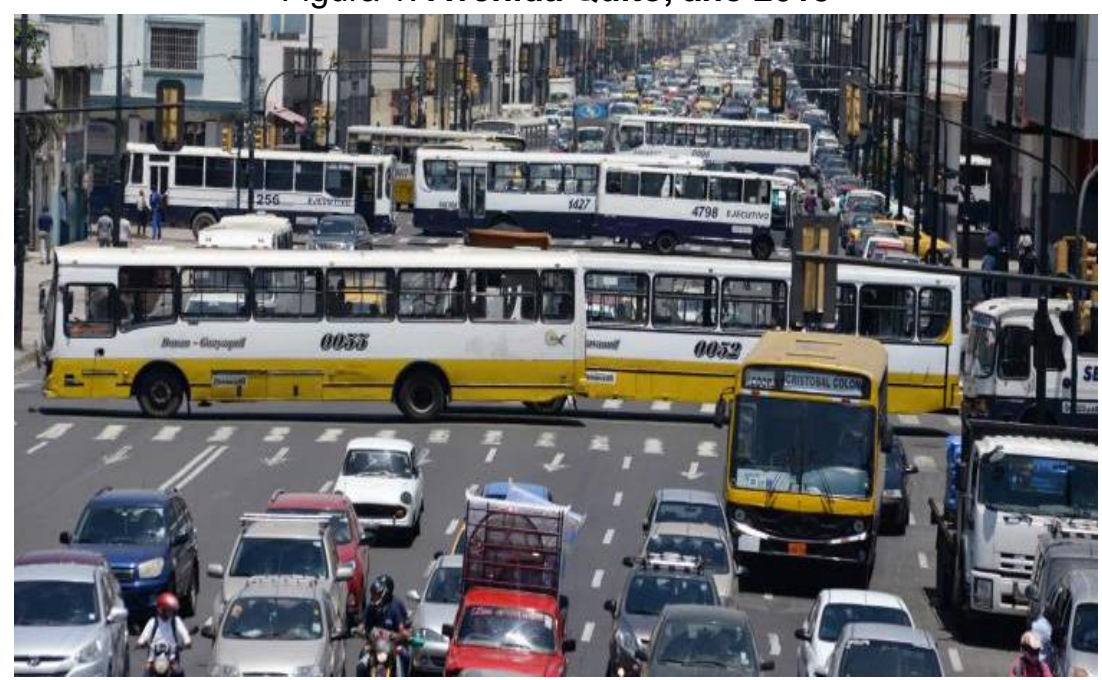

Fuente: Diario El Universo

En el año 2017 la actual Autoridad de Tránsito Municipal (ATM) inauguró el primer corredor de buses de transporte público con 70 unidades las que circulan por un corredor de 104 paraderos señalizados.

En este primer circuito, 70 unidades darán el servicio a la comunidad, los pasajeros subirán por la parte delantera y bajarán utilizando la puerta trasera.

El propósito de la entidad que regula el tránsito en Guayaquil es reordenar el sistema de transporte público convencional y para ello se prevé la implementación de quince corredores.

Citación: NARANJO RAMOS, Y. y ARELLANO, B. La Metrovía como sistema BRT - el caso de la ciudad de Guayaquil. En: Libro de proceedings, CTV 2018. XII Congreso Internacional Ciudad y Territorio Virtual. "Ciudades y Territorios Inteligentes". UNCuyo, Mendoza, 5-7 septiembre 2018. Barcelona: CPSV, 2018, p. 129-142. 
Figura 2. Recorrido del primer corredor vial centro, año 2017

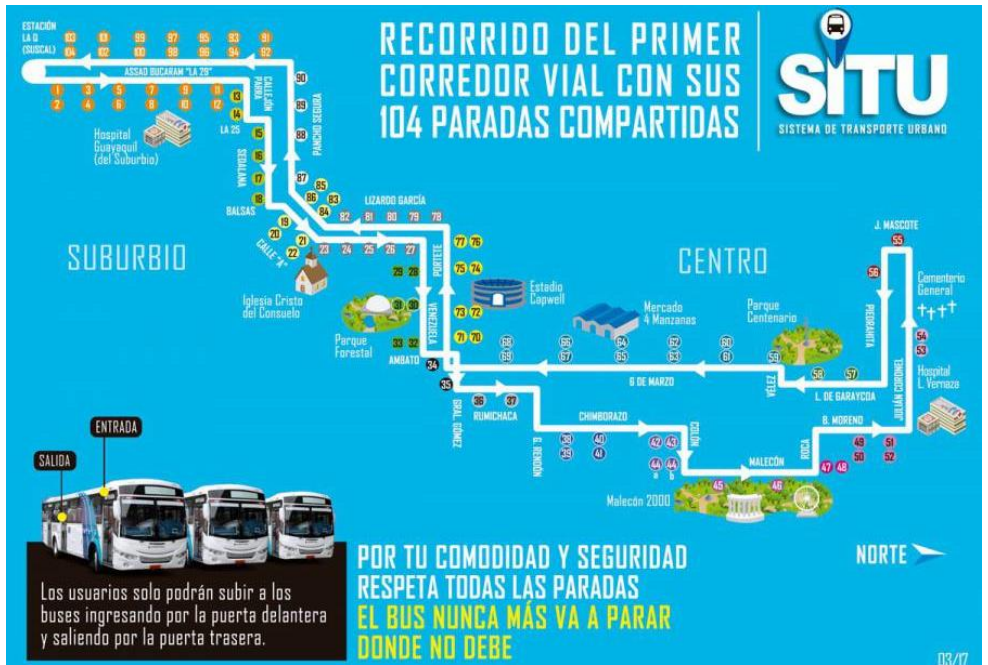

Fuente: Diario El Universo

\section{- Vehículo privado (cuenta propia)}

Según datos de la ATM, en el año 2016 se matricularon aproximadamente 334 mil vehículos en la ciudad de un parque automotor estimado de 340 mil. Asimismo el crecimiento en cuanto a la adquisición de nuevos vehículos en la ciudad, en el año 2017 se vendió 1726 unidades más que en el año 2016.

\section{Tabla 1. Comportamiento en el mercado automotriz hasta el año 2017}

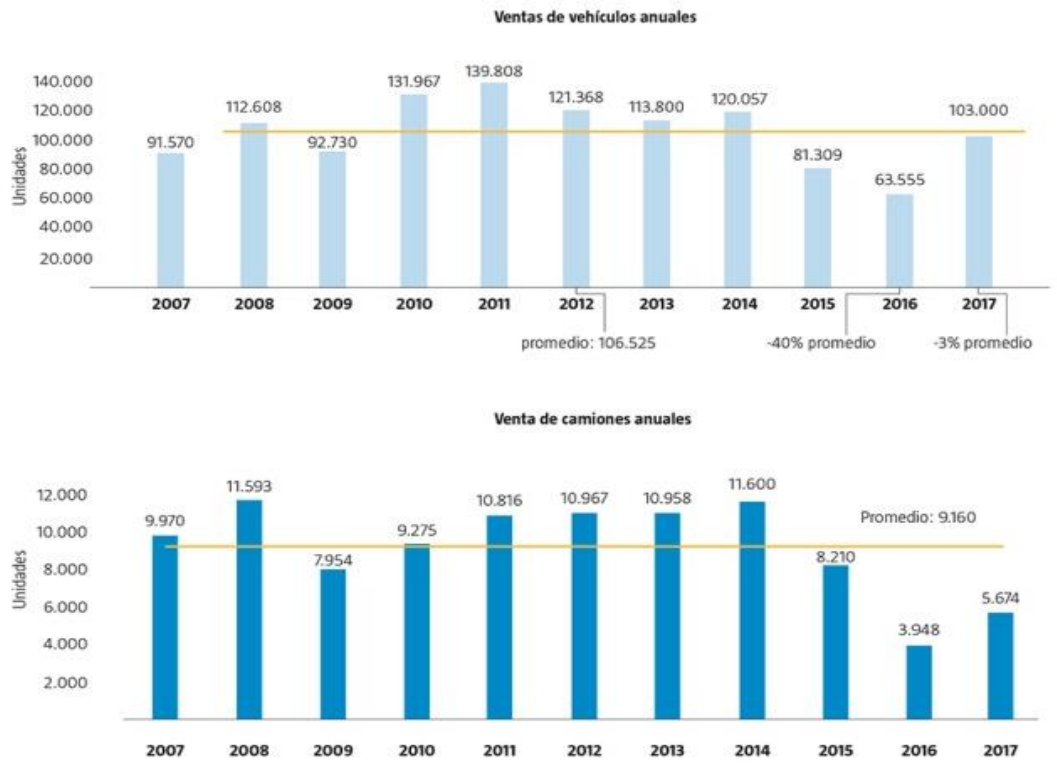

Fuente: INEC, 2015

- Transporte comercial

- Taxi

En la ciudad actualmente el taxi amarillo ejecutivo o privado que no necesariamente son de color amarillo y están regulados, son parte del modo de transporte y además uno de los más

Citación: NARANJO RAMOS, Y. y ARELLANO, B. La Metrovía como sistema BRT - el caso de la ciudad de Guayaquil. En: Libro de proceedings, CTV 2018. XII Congreso Internacional Ciudad y Territorio Virtual. "Ciudades y Territorios Inteligentes". UNCuyo, Mendoza, 5-7 septiembre 2018. Barcelona: CPSV, 2018, p. 129-142. 
utilizados, ya que el usuario en muchas ocasiones prefiere un uso exclusivo y una prioridad al momento de trasladarse.

Figura 3. Vía principal, medios de transporte comercial, público y privado

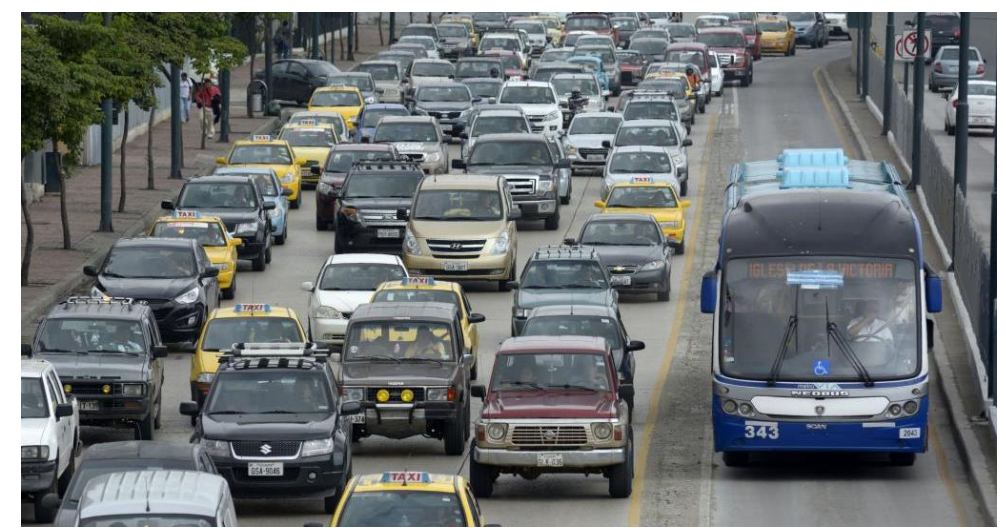

Fuente: Naranjo, 2018

\subsection{El sistema de transporte público masivo}

Es el Sistema actual Integrado de Transporte Masivo Urbano de la ciudad de Guayaquil, desarrollado por la Municipalidad de Guayaquil, se basa en un modelo BRT (Bus Rapid Transit), que cuenta con los siguientes elementos básicos:

- Vías exclusivas separadas del resto del tránsito.

- Estaciones de transferencia.

- Centro de control operacional.

- Pago antes de embarcar.

- Paradas elevadas de acceso controlado.

Figura 4. Plano Troncales Metrovía Guayaquil

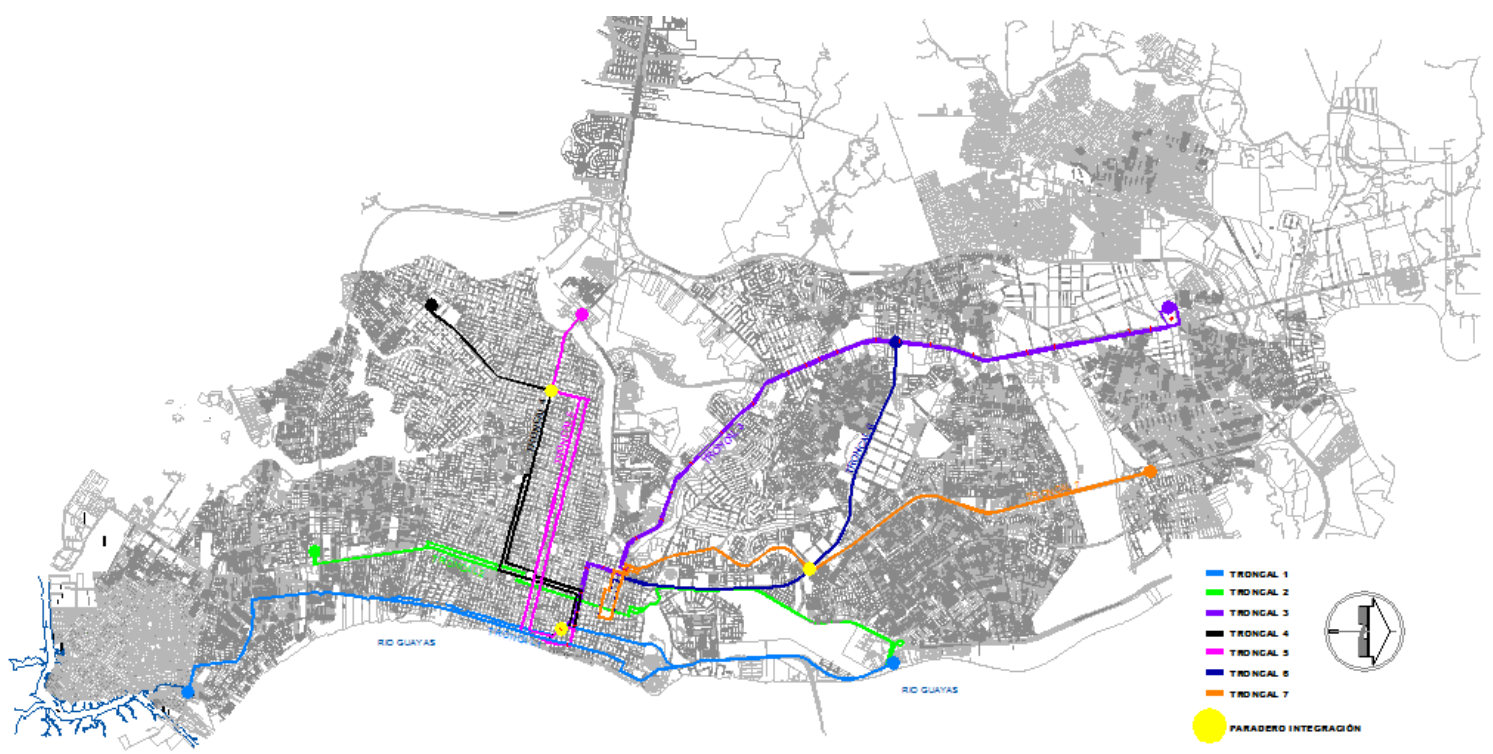

Fuente: Huerta, 2012

Citación: NARANJO RAMOS, Y. y ARELLANO, B. La Metrovía como sistema BRT - el caso de la ciudad de Guayaquil. En: Libro de proceedings, CTV 2018. XII Congreso Internacional Ciudad y Territorio Virtual. "Ciudades y Territorios Inteligentes". UNCuyo, Mendoza, 5-7 septiembre 2018. Barcelona: CPSV, 2018, p. 129-142. 
Consiste en una serie de buses articulados y alimentadores que transitan por la ciudad por carriles predestinados, transportando pasajeros y dejándolos en una serie de paradas ubicadas aproximadamente a 300 metros una de otras.

Existen 3 tipos de estaciones, cada una de estas tiene formas muy particulares de acuerdo a su uso, cantidad de pasajeros y número de buses articulados que se conectan a ella.

\section{Estaciones tipo caseta:}

Constan de un techo en forma de " $\mathrm{V}$ " invertida, suelen ser las más comunes, son pequeñas, en algunos casos solo tiene un acceso por donde entran y salen los pasajeros.

Figura 5. Parada tipo caseta, calle Machala, 2018

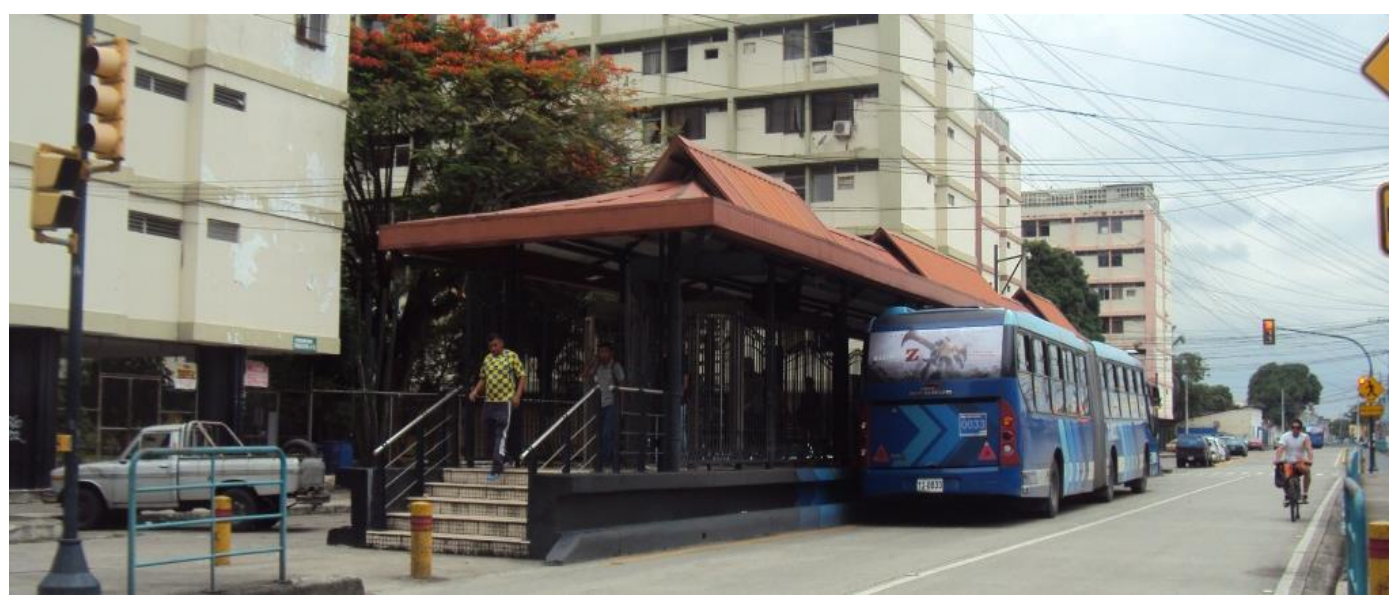

Fuente: Naranjo, 2018

Estaciones tipo paso peatonal: Se trata de una idea innovadora en la que los pasajeros pueden acceder a las estaciones por medio de un paso peatonal elevado, el mismo que en ciertos casos contienen locales comerciales.

Figura 6. Parada Universidad Católica de Santiago de Guayaquil

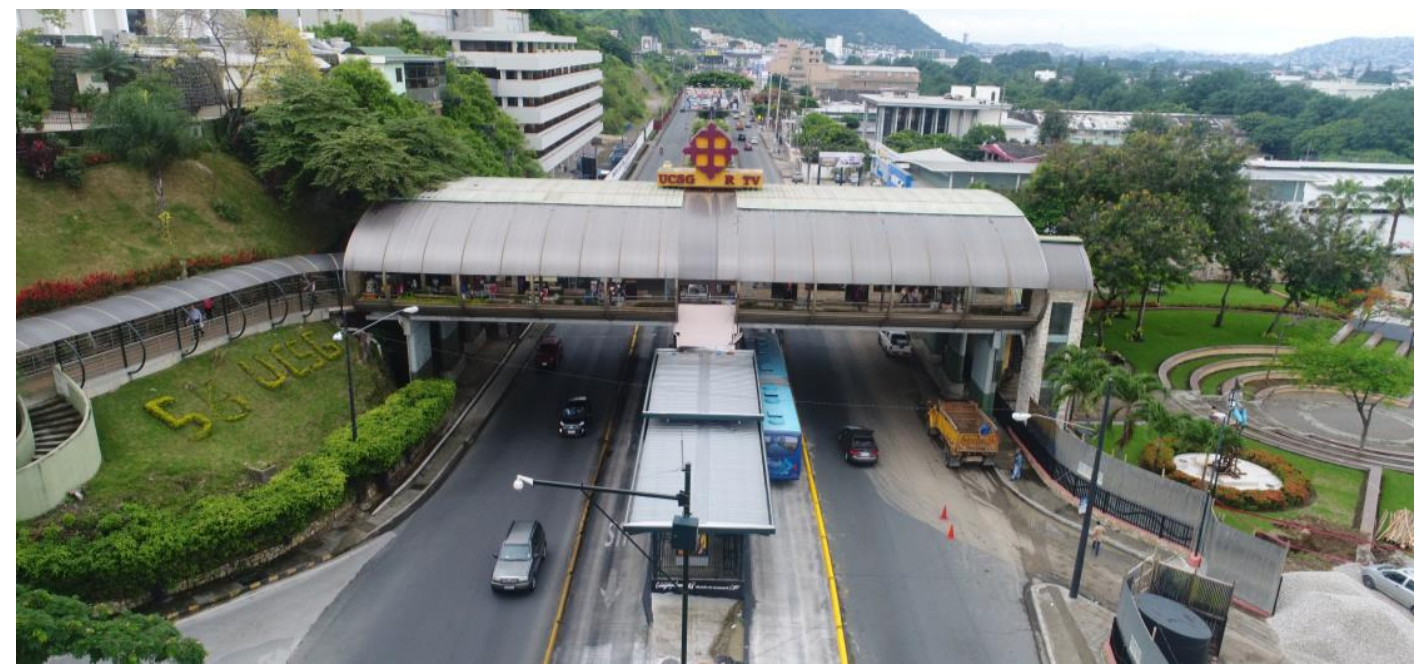

Fuente: Fotografía aérea (Naranjo, 2018)

Citación: NARANJO RAMOS, Y. y ARELLANO, B. La Metrovía como sistema BRT - el caso de la ciudad de Guayaquil. En: Libro de proceedings, CTV 2018. XII Congreso Internacional Ciudad y Territorio Virtual. "Ciudades y Territorios Inteligentes". UNCuyo, Mendoza, 5-7 septiembre 2018. Barcelona: CPSV, 2018, p. 129-142. 
Estaciones de integración: Son las estructuras más grandes en relación a las otras paradas, albergan gran cantidad de pasajeros y sirven como base de llegada y salida de los buses articulados.

\section{Figura 7. Estación de integración, Río Daule}

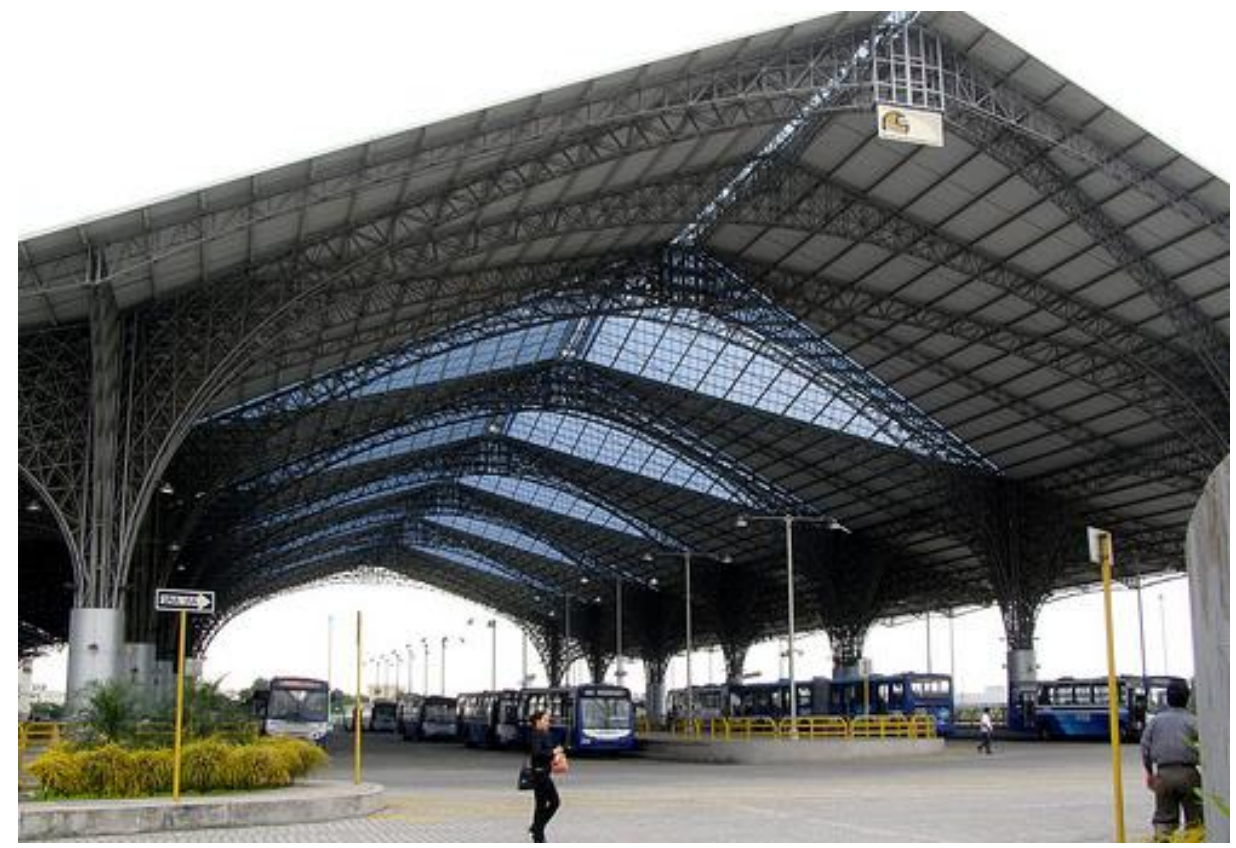

Fuente: Naranjo, 2018

\subsubsection{Estructura del sistema}

Constituida por tres líneas troncales, cuyos buses circulan en carriles para su uso exclusivo, estos carriles permiten que las personas que viajan en bus tengan preferencia en la circulación y por lo tanto lleguen más rápido a su destino.

A lo largo de la vía existen estaciones cerradas con cubiertas para proteger a los pasajeros del sol y de la lluvia. Para ingresar las personas primero deben cancelar su pasaje y luego abordar el bus en forma cómoda y rápida. Las personas por lo tanto no tienen que pagar al chofer del bus, sino a su entrada de las estaciones las mismas que se denominan de pre-embarque.

En los extremos de cada tramo se encuentran grandes estaciones a donde llegan todos los buses tanto de la línea troncal, como de los buses que van a los barrios y que se denominan "buses alimentadores". Las personas podrán utilizar libremente, sin pagar otro pasaje, cualquier bus que se encuentre dentro de la terminal. (Huerta, 2007).

\subsubsection{Primera Troncal "Terminal El Guasmo - Terminal Río Daule”, Julio 2006}

La primera línea troncal o primer corredor corresponde a la línea que se extiende entre las terminales de transferencia "El Guasmo" y "Río Daule", con una longitud (ida y vuelta) de 31,59 kilómetros (ver figura 8). 


\section{Libro de proceedings \\ Territorios Inteligentes \\ ISBN: 978-84-8157-661-0

Figura 8. Troncal 1. Estación Río Daule - Terminal Guasmo

RUTA DE METROVÍA

TRONCAL 1

Estación Río Daule - Terminal Guasmo

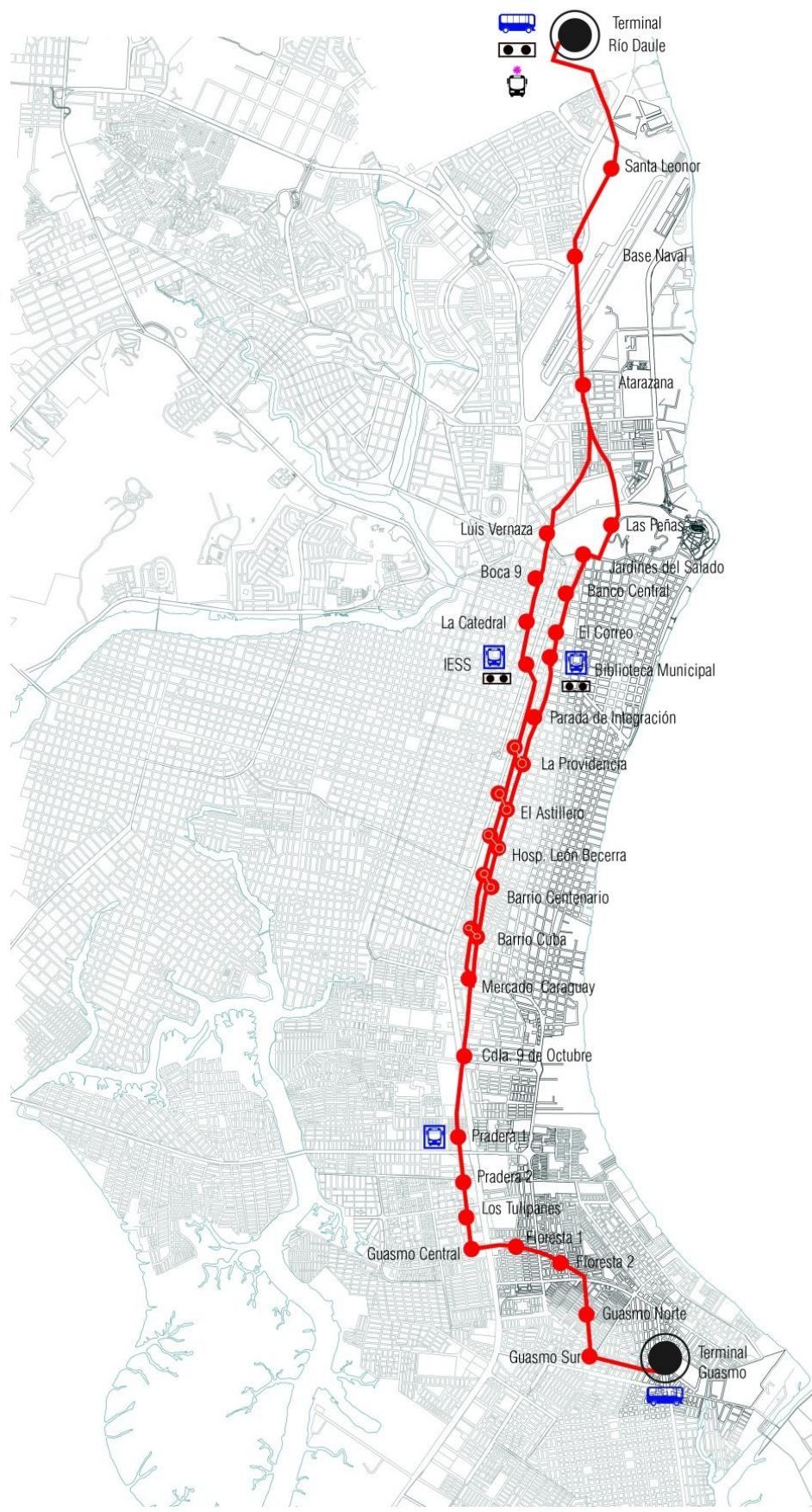

\subsubsection{Segunda troncal "Terminal 25 de Julio - Terminal Río Daule"}

La segunda troncal se denomina Terminal 25 de Julio - Terminal Río Daule (Ver figura 9), inicia desde el intercambiador de tráfico ubicado en la intersección de la Vía Perimetral y Avenida 25 de Julio y culmina en la Terminal Río Daule. 
Esta troncal tiene una extensión aproximada (ida y vuelta) de 42,63 kilómetros en carriles dobles con 23 paradas de pasajeros más 4 paradas que permiten combinación, sumando 27 paradas en esta troncal.

Figura 9. Troncal 2. Estación Río Daule - Terminal 25 de Julio

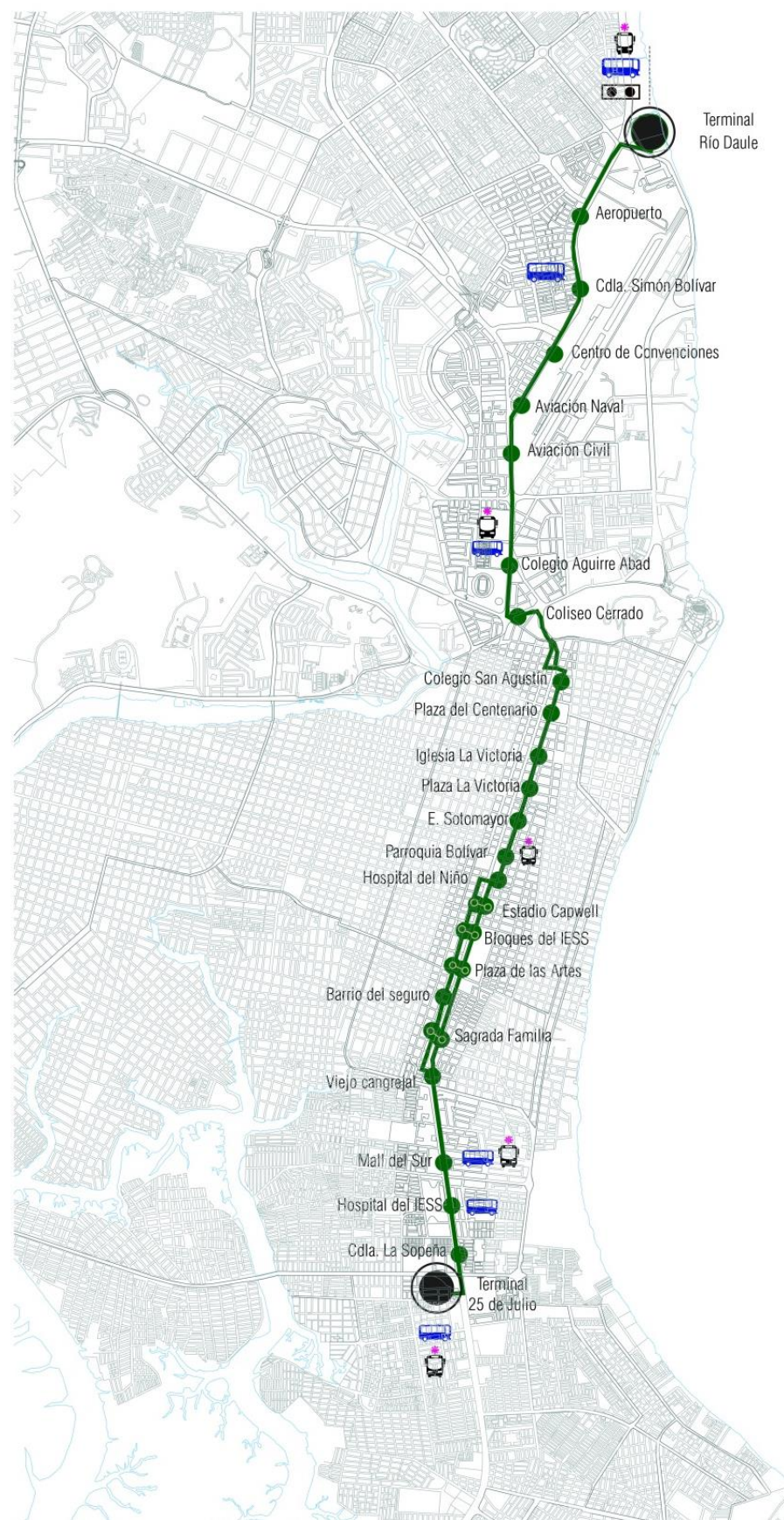

RUTA DE METROVÍA

Estación Río Daule - Terminal 25 de Julio TRONCAL 2

Fuente: Naranjo, 2018

\subsubsection{Tercera troncal "Terminal Bastión Popular - Centro”, abril 2008}

La tercera troncal denominada Terminal Bastión Popular - Centro, tiene una extensión aproximada de 31,49 kilómetros con 24 paradas de pasajeros a lo largo de su recorrido (ver figura 10).

Citación: NARANJO RAMOS, Y. y ARELLANO, B. La Metrovía como sistema BRT - el caso de la ciudad de Guayaquil. En: Libro de proceedings, CTV 2018. XII Congreso Internacional Ciudad y Territorio Virtual. "Ciudades y Territorios Inteligentes". UNCuyo, Mendoza, 5-7 septiembre 2018. Barcelona: CPSV, 2018, p. 129-142. 


\section{Libro de proceedings \\ Territorios Inteligentes \\ ISBN: 978-84-8157-661-0

Figura 10. Troncal 3. Terminal Bastión popular

RUTA DE METROVÍA

TRONCAL 3

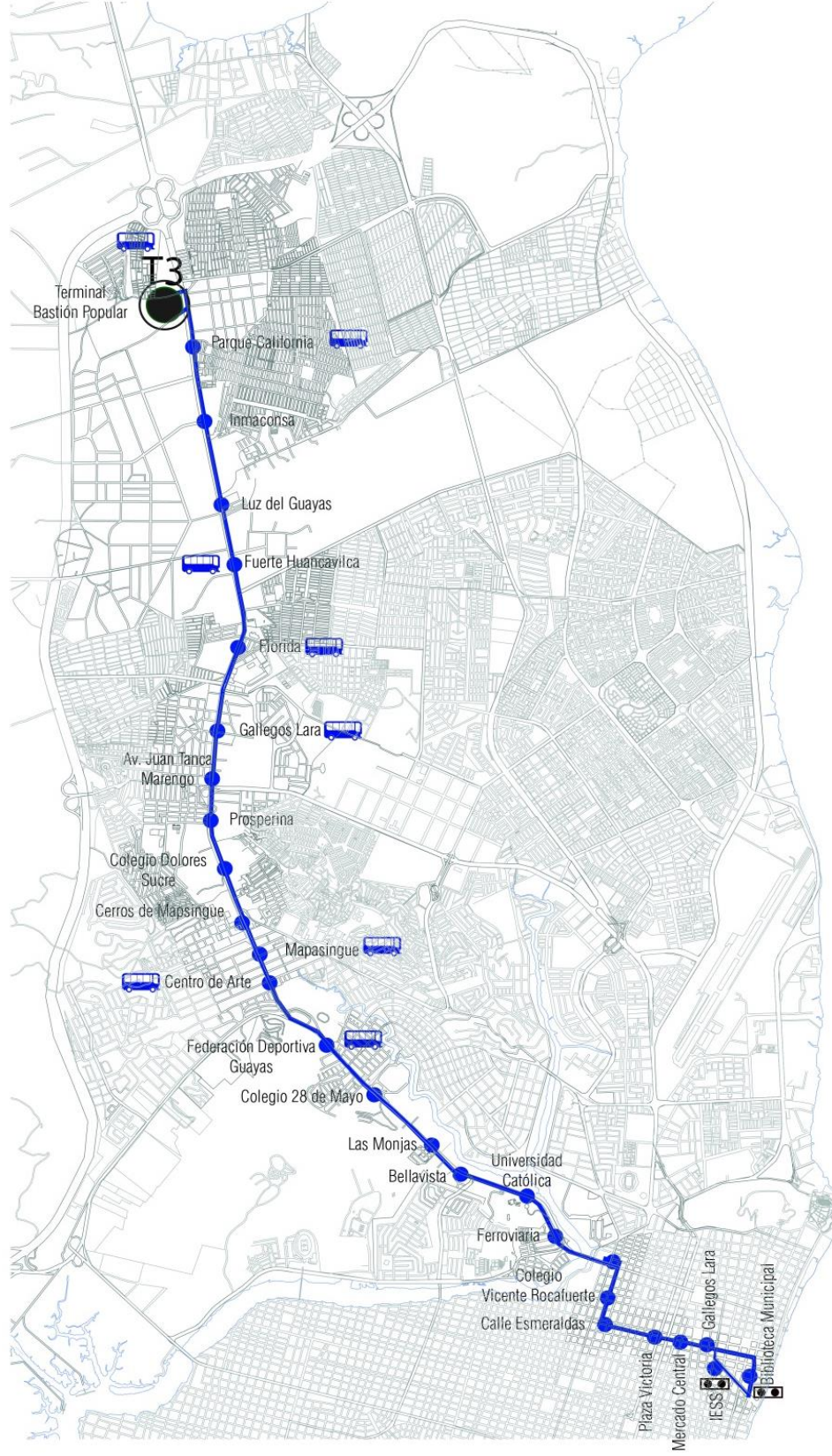

Terminal Bastión Popular

Fuente: Naranjo, 2018

Las ciudades se configuran en los lugares donde las personas viven, trabajan y desarrollan sus actividades, ya sea dentro o fuera de los hogares. Las actividades que se realizan fuera de casa demandan formas diferentes de desplazamientos: caminata, transporte mecanizado (bicicleta) o motorizado (autobuses, motocicletas, automóviles y metro).

Para entender cómo se realizan estos desplazamientos y el tipo de transporte para llevarlos a cabo, es necesario comprender cómo se estructura la ciudad y cuáles son los factores de mayor influencia en la movilidad de las personas y en la elección de los modos de transporte (Alcântara V, 2010).

Citación: NARANJO RAMOS, Y. y ARELLANO, B. La Metrovía como sistema BRT - el caso de la ciudad de Guayaquil. En: Libro de proceedings, CTV 2018. XII Congreso Internacional Ciudad y Territorio Virtual. "Ciudades y Territorios Inteligentes". UNCuyo, Mendoza, 5-7 septiembre 2018. Barcelona: CPSV, 2018, p. 129-142. 
Guayaquil posee actualmente una estructura urbana donde se relacionan diferentes actividades, las mismas que permiten estudiar dicha relación, principalmente las redes de servicio y movilidad que se dan alrededor del sistema de transporte público masivo. La modalidad del sistema de transporte masivo bajo principios del BRT, son propuestas universales que se han implementado en las ciudades de Latinoamérica principalmente, en Guayaquil es un sistema troncalizado integrado unilateralmente, que pretende cubrir las necesidades de movilidad de la ciudad.

Sin lugar a dudas se ha generado un cambio en la estructura urbana de la ciudad ya que las calles donde se implementan los ejes de este sistema en algunos casos se han cerrado al tráfico vehicular privado y en otros casos se ha compartido. Se debe constituir como un sistema ya que son troncales que en este caso de las 7 deben implementarse al menos 5 , sin embargo, actualmente solo funcionan 3 , la principal característica es que funcione sin interrupciones sobre un carril propio exclusivo, y en casos excepcionales tenerlo compartido, esto se da ya que la misma trama urbana de Guayaquil no permite tener un carril rápido y exclusivo.

El sistema de transporte público masivo no se ha desarrollo como proyecto integral de movilidad ya que no genera cambios modales en sus ejes de conexión de parada:

- La ciudad no cuenta con carril de bicicleta.

- Sólo se establece señalización a nivel de calle para el paso peatonal.

- Las aceras libres solo para uso peatonal son de no más de un $1 \mathrm{~m}$, ya que el resto es utilizado para iluminación, área verde y demás elementos.

- No existe la opción de dejar el vehículo en un área destinada y conectarse con el transporte en general.

Finalmente se puede decir que existe actualmente un cambio en la ciudad ya que las personas se transportan en un menor tiempo, a bajo costo y en un carril exclusivo, sin embargo, el tiempo de espera no se respeta y la cantidad de buses no abastece a la población.

\section{Bibliografía}

ALCÂNTARA V, E. Análisis de la movilidad urbana. Septiembre de 2010. Disponible en: https://www.caf.com/media/3155/An\%C3\%A1lisis movilidad urbana.pdf

DE GRANGE C, L. El gran impacto del metro. En: EURE [en línea] 36 (107): 125-131. 2010. Santiago. DOI: http://dx.doi.org/10.4067/S0250-71612010000100007

DREHER A, D. Experiencia Guayaquil: Regeneración Urbana. La Ciudad viva como URBS, Quito. 2009. (pág. 1).

ESTRADA ICAZA, J. Notas de un viaje de cuatro siglos, Guía Historica de Guayaquil. Tomo 1. Guayaquil: Poligrafica. 1995.

HOYOS G, M. Los Recuerdos de la Iguana, Hisorias del Guayaquil que se fue. Guayaquil. 2008.

ROGAT, J. Planificacion e implementacion de un sistema de Bus Rapido en America Latina. [en línea] Unep Riso Centre, 19-25. 2009. Disponible en: http://orbit.dtu.dk/files/4045483/Rogat2.pdf

VEGA, J. \& DÍAZ, E. Estudio socioeeconomico de los usuarios de metrovía en la ciudad de Guayaquil y medición del Impacto de la Publicidad en el segmento. [en línea] 2012. Disponible en: https://dspace.ups.edu.ec/bitstream/123456789/3328/1/UPS-GT000352.pdf

Citación: NARANJO RAMOS, Y. y ARELLANO, B. La Metrovía como sistema BRT - el caso de la ciudad de Guayaquil. En: Libro de proceedings, CTV 2018. XII Congreso Internacional Ciudad y Territorio Virtual. "Ciudades y Territorios Inteligentes". UNCuyo, Mendoza, 5-7 septiembre 2018. Barcelona: CPSV, 2018, p. 129-142. 